\title{
DNA methylome signatures as epigenetic biomarkers of hexanal associated with lung toxicity
}

\author{
Yoon Cho ${ }^{1}$, Mi-Kyung Song ${ }^{2}$, Jae-Chun Ryu ${ }^{\text {Corresp. } 1}$ \\ ${ }^{1}$ Korea Institute of Science and Technology, Seoul, Republic of Korea \\ 2 Korea Institute of Toxicology, Jeongeup, Republic of Korea \\ Corresponding Author: Jae-Chun Ryu \\ Email address: yooncho@kist.re.kr
}

Background. Numerous studies have investigated the relationship of environmental exposure, epigenetic effects, and human diseases. These linkages may contribute to the potential toxicity mechanisms of environmental chemicals. Here, we investigated the epigenetic pulmonary response of hexanal, a major indoor irritant, following inhalation exposure in F-344 rats. Methods. Based on DNA methylation profiling in gene promoter regions, we identified hexanal-characterized methylated sites and target genes using an unpaired t-test with a fold-change cutoff of $\geq 3.0$ and a $p$-value $<0.05$. We also conducted an integrated analysis of DNA methylation and mRNA expression data to identify core anticorrelated target genes of hexanal exposure. To further investigate the potential key biological processes and pathways of core DNA methylated target genes, Gene Ontology and Kyoto Encyclopedia of Genes and Genomes pathway enrichment analysis were performed. Results. 36 dose-dependent methylated genes and anti-correlated target genes of DNA methylation and mRNA in lung tissue of hexanal exposed F-344 rats were identified. These genes were involved in diverse biological processes such as neuroactive ligand-receptor interaction, protein kinase cascade, and intracellular signaling cascade associated with pulmonary toxicity. These results suggest that novel DNA methylationbased epigenetic biomarkers of exposure to hexanal and elucidate the potential pulmonary toxicological mechanisms of action of hexanal. 


\section{DNA Methylome Signatures as Epigenetic Biomarkers 2 of Hexanal Associated with Lung Toxicity}

3 39
Yoon Cho ${ }^{1}$, Mi-Kyung Song ${ }^{2}$, Jae-Chun Ryu ${ }^{1}$

${ }^{1}$ Korea Institute of Science and Technology, Seoul, Republic of Korea

${ }^{2}$ Korea Institute of Toxicology, Jeongeup, Republic of Korea

Corresponding Author:

Jae-Chun Ryu ${ }^{1}$

5 Hwarang-ro 14-gil, Seongbuk-gu, Seoul, 02792, Korea

Email address: ryujc@kist.re.kr

\section{Abstract}

\section{Background.}

Numerous studies have investigated the relationship of environmental exposure, epigenetic effects, and human diseases. These linkages may contribute to the potential toxicity mechanisms of environmental chemicals. Here, we investigated the epigenetic pulmonary response of hexanal, a major indoor irritant, following inhalation exposure in F-344 rats.

\section{Methods.}

Based on DNA methylation profiling in gene promoter regions, we identified hexanalcharacterized methylated sites and target genes using an unpaired $t$-test with a fold-change cutoff of $\geq 3.0$ and a $p$-value $<0.05$. We also conducted an integrated analysis of DNA methylation and mRNA expression data to identify core anti-correlated target genes of hexanal exposure. To further investigate the potential key biological processes and pathways of core DNA methylated target genes, Gene Ontology and Kyoto Encyclopedia of Genes and Genomes pathway enrichment analysis were performed.

\section{Results.}

36 dose-dependent methylated genes and anti-correlated target genes of DNA methylation and mRNA in lung tissue of hexanal exposed F-344 rats were identified. These genes were involved in diverse biological processes such as neuroactive ligand-receptor interaction, protein kinase cascade, and intracellular signaling cascade associated with pulmonary toxicity.

These results suggest that novel DNA methylation-based epigenetic biomarkers of exposure to hexanal and elucidate the potential pulmonary toxicological mechanisms of action of hexanal. 


\section{Introduction}

41 The role of epigenetics has been expanded to the field of environmental toxicology to include

42 exposure to chemical agents and pathogenesis of diseases (Watson \& Goodman, 2002; Szyf, 2011). It is defined as environmental epigenetics (Ho et al., 2012) and provides important insights into the linkage between environmental exposure and human health based on toxicogenomic concepts (Burris \& Baccarelli, 2014; Reamon-Buettner et al., 2008). The implication of environmental epigenetics in toxicogenomics has been demonstrated in numerous studies. It may provide the cellular and molecular signatures affected by exposure to environmental factors and contribute to understanding epigenetic toxicological mechanisms (Baccarelli, 2009). This approach is used for developing exposure biomarkers for detecting the response at low doses, early effects and elucidating the underlying modes of action for environmental disease (McHale et al., 2010). Therefore, it has been considered an effective strategy for toxicological risk assessment of environmental chemicals.

Exposure to a variety of environmental factors induces epigenetic alterations which emerge as key factors of numerous important cellular processes including regulation in gene expression. Also, aberrant epigenetic patterns are critical for the development of diseases and cancer progression (Zoghbi \& Beaudet, 2016; Kagohara et al., 2018; Koh \& Hwang, 2019).

Furthermore, recent studies have highlighted the importance of epigenetic biomarkers such as miRNA and DNA methylation-based biomarkers. Epigenetic biomarkers are emerging as screening tools for exposure and risk assessments of environmental chemicals (Ray et al., 2014). However, the use of epigenetic changes as a predictive exposure biomarker for exposure to environmental toxicants remains unclear. Here, we aimed to identify the epigenetic biomarkers of hexanal (hexaldehyde) for exposure and risk assessment based on the DNA methylome signature.

Hexanal is one among the aldehydes which are classified as microbial volatile organic compounds (mVOCs). mVOCs are emitted during metabolism in micro-organisms, including fungi and bacteria. It is known that mVOCs are highly abundant in the indoor environment (Korpi et al., 2009). Previous studies demonstrated that exposure to mVOCs may induce diverse adverse health effects such as irritation of the respiratory tract and eyes and inflammatory responses (Korpi et al., 2009; Thorn \& Greenman, 2012). Of the more than 1,000 compounds of mVOCs, aldehydes are a predominant group (Garcia-Alcega et al., 2018). However, the toxicological data of mVOCs using omics technologies is still not well understood. We previously investigated the toxicogenomic response of hexanal, an important indoor air pollutant, using an in vitro system (Cho et al., 2014; 2015). In this study, we aimed to investigate the epigenetic response based on DNA methylation of hexanal exposure using the in vivo model system.

To clarify the DNA methylation networks by exposure to hexanal associated with lung toxicity, we analyzed the DNA methylation profiling of lung tissues of F-344 rats following inhalation exposure to hexanal. In the three hexanal inhalation exposure groups $(600,1,000$, and 1,500 
$79 \mathrm{ppm})$, the expression of 73 methylated genes was altered and 36 dose-dependent methylated

80 genes were also identified using a 3.0-fold change cut-off and p-value $<0.05$.

81 To further investigate the effect of hexanal exposure on DNA methylation and gene expression

82 profiles, we conducted an integrated analysis of the DNA methylation and mRNA expression

83 profiles. Core anti-correlated genes which are involved in key biological processes associated

84 with pulmonary toxicity were identified. These results provide that a novel epigenetic biomarker

85 of exposure to hexanal and potential important quantitative biomarkers for risk assessments. This

86 approach of DNA methylation-environmental factors may also reveals new mechanistic insights

87 on the epigenetic actions of pulmonary toxicity.

88

89 Materials \& Methods

90 Vertebrate Animal Study

91 Test animal

92 Forty male and female Fischer 344 rats of both sexes (10 rats/group), 7 weeks of age, were

93 purchased from ORIENT BIO INC. (Seongnam, Korea). Prior to the experiment, animals were

94 housed in stainless-steel cages $(255 \mathrm{~W} \times 3465 \mathrm{~L} \times 3200 \mathrm{H} \mathrm{mm})$ and acclimated for 5 days.

95 Purification and quarantine periods were 3 or less, and during pretest and exposure periods, 2 or

96 less were kept in stainless-steel cages. During the acclimation period, all animals are observed

97 once a day to see clinical symptoms caused by the disease. Animals with diseases or

98 abnormalities observed on physical examination are euthanized through $\mathrm{CO}_{2}$ inhalation. Animal

99 rooms had a 12-h light/dark cycle and controlled temperature $\left(22 \pm 3{ }^{\circ} \mathrm{C}\right)$ and humidity $(30-70 \%)$.

100 All animals were given a sterilized commercial pellet diet (PMI Nutrition International, USA)

101 and sterilized water. All experimental procedures were approved by the Institutional Animal

102 Care and Use Committee (IACUC) of Korea Institute of Toxicology (IACUC No. 1311-0301).

103

104

\section{Clinical, biochemical and histopathological examinations}

105 Test animals were subjected to examine every day for any clinical, blood biochemical and

106

107

108

109

110

111

112

113

114

115

116

117

118 histopathological symptoms and mortality. Total body weight was measured twice a week during the 4 weeks exposure period. Test animals surviving to the end of the exposure period received completed necropsy. Test animals were euthanized using isoflurane anesthesia. For autopsy animals, gross autopsy findings were observed before organ weight measurement. Whole blood (WB) was rapidly collected for blood biochemical analysis from the abdominal aorta under isoflurane. Serum was obtained from WB by centrifuging at 3,000 $\mathrm{x} \mathrm{rpm}$ for 10 minutes at room temperature and analyzed for AST(Aspartate aminotransferase), ALT(Alanine aminotransferase), ALP (Alkaline phosphatase), CK((Creatine phosphokinase), GLU (Glucose), TP (Total protein), ALB (Albumin), GLO (Globulin), A/G (Albumin/globulin ratio), BUN (Blood urea nitrogen), CREA (Creatinine), TG (Triglyceride), PL (Phospholipid), TCHO (Total cholesterol), TBIL (Total bilirubin), GGT (Gamma glutamyl transferase), $\mathrm{Ca}$ (Calcium), $\mathrm{IP}$ (Inorganic phosphorus), $\mathrm{Cl}$ (Chloride), $\mathrm{Na}$ (Sodium) and $\mathrm{K}$ (Potassium) using an autochemical analyzer, Toshiba 120FR NEO(Toshiba Co., Japan). The lung tissues were collected from all

Peer] reviewing PDF | (2020:07:51029:1:1:CHECK 2 Dec 2020) 
119 animals and preserved in 10\% neutral buffered formalin and embedded with paraffin wax.

120 Tissues were stained with hematoxylin and eosin (H\&E) (Cho et al., 2016; 2017).

121

122 Exposure design

123 All animal experiments were carried in accordance with relevant guidelines and regulations.

124 Exposure experiments were designed following the OECD guideline for the testing of chemicals

125 No. 412 "Subacute Inhalation Toxicity" (OECD, 2009), considering animal welfare. Hexanal

126 vapor was generated with a bubbling generator and animals were exposed to it inside a flow-past

127 nose-only inhalation chamber. Hexanal exposure concentrations were at target levels of 600,

1281,000 , and 1,500 ppm, and the control group was exposed to filtered clean air. Grouped animals

129 had a pre-exposure period of about 2 days before exposure began. During the pre-exposure

130 period, holder adaptation training was performed in accordance with the standard operation

131 procedure to reduce stress caused by non-inhalational exposure. Residual animals excluded from

132 the test were euthanized with $\mathrm{CO}_{2}$. The animals (10 rats per group) were exposed to hexanal for

1334 weeks ( $4 \mathrm{~h} /$ day, 5 days/week) in the nose-only inhalation chamber. Using a GC-FID

134 (SHIMADZU, Japan), exposure concentration of hexanal vapor was measured thrice daily. We

135 also monitored the environment in the inhalation chamber such as chamber flow rate,

136 temperature $\left({ }^{\circ} \mathrm{C}\right)$, relative humidity $(\%)$, chamber pressure (-Pa) and oxygen concentration $(\%)$

137 more than 4 times during the exposure period (Cho et al., 2016; 2017).

138

139

\section{DNA preparation}

140 Genomic DNA was isolated from the homogenized lung tissue of rats, and only the supernatant

141 was used for extraction. DNA samples of 6 rats from each group (control, low-dose, middle-

142 dose, and high-dose group; a total of 24 DNA samples) were used for the microarray analysis for

143 all 40 rats used in the study. Using Qiagen's QIAamp DNA Mini kit (QIAGEN, Hilden,

144 Germany), genomic DNA was extracted as described in our previous study (Cho et al., 2018).

145 The genomic DNA purity and concentration were measured using ND-1000 spectrophotometer

146 (NanoDrop Technologies, Wilmington, DE) and electrophoresis conducted in a 1.5\% agarose gel

147 in $1 \mathrm{X}$ TAE buffer ( $4.8 \mathrm{~g}$ of Tris, $1.14 \mathrm{~mL}$ of acetic acid, $2 \mathrm{~mL}$ of $0.5 \mathrm{M}$ EDTA at $\mathrm{pH} 8.0$, and

148 ethidium bromide) at a constant $100 \mathrm{~V}$ for $15 \mathrm{~min}$.

149

150 Fragmentation of DNA

151 To extract only methylated DNA, the genomic DNA size should be about $200 \mathrm{bp}$ to $1,000 \mathrm{bp}$.

152 Therefore, genomic DNA was fragmented into $200 \mathrm{bp}$ to 1,000 bp sections using a Sonic

153 Dismembrator 550 (Fisher Scientific, USA) with 3 cycles comprising 4 cycles of $20 \mathrm{sec}$ 'ON' and

1541 cycle of $20 \mathrm{sec}$ 'OFF'. To determine the size of the fragmented DNA, agarose gel

155 electrophoresis and ethidium bromide staining were performed using DNA size markers of 500-

15610,000 base pairs in size.

157

158 Methylated DNA immunoprecipitation (MeDIP) 
159

160

161

162

163

164

165

166

167

168

169

170

171

172

173

174

175

176

177

178

179

180

181

182

183

184

185

186

187

188

189

190

191

192

193

194

195

196

197

198

As described in our previous study (Cho et al., 2018), MeDIP was performed with MethylMiner Methylated DNA Enrichment Kit (Invitrogen, Carlsbad, CA, USA) according to the manufacturer's instructions. Fragmented DNA $1 \mu \mathrm{g}$ and untreated control DNA (Input) $3 \mu \mathrm{g}$ were used for quality and labelling procedures. Briefly, Dynabeads M-280 Streptavidin $10 \mu 1$ was combined with $7 \mu \mathrm{l}$ of MBD (methyl-CpG binding domain)-Biotin Protein. The MBD-magnetic bead conjugates were washed thrice and resuspended in 1 volume of $1 \mathrm{X}$ bind/wash buffer. The capture reaction was conducted by adding of $1 \mu \mathrm{g}$ sonicated DNA to the MBD magnetic beads on a rotating mixer for $1 \mathrm{~h}$ at room temperature. Next, the beads were washed three times with 1 $\mathrm{X}$ bind/wash buffer. The methylated DNA was eluted as a single fraction with a high-salt elution buffer $(2,000 \mathrm{mM} \mathrm{NaCl})$. Consequently, each fraction was concentrated by ethanol precipitation using $1 \mu \mathrm{L}$ glycogen $(20 \mu \mathrm{g} / \mu \mathrm{L}), 1 / 10$ th volume of $3 \mathrm{M}$ sodium acetate ( $\mathrm{pH} 5.2)$, and two volumes of $100 \%$ ethanol, and then resuspended in $60 \mu \mathrm{L}$ of DNase-free water. The eluted methylated DNA immunoprecipitation samples were stored at $-20{ }^{\circ} \mathrm{C}$ until further use. This experiment protocol was referred from out previous research in Cho et al. (2018).

\section{Epigenome-wide DNA methylation}

First, whole genome amplification kit (GenomePlex Complete Whole Genome Amplification Kit, SIGMA-ALDRICH, USA) was used to amplify DNA and methylated immunoprecipitation (IP) samples according to the manufacturer's instructions. The amplified samples were purified using the QIAQuick PCR clean-up kit (QIAGEN, Hilden, Germany). The amplified DNA and 4 $\mu \mathrm{g}$ of the methylated IP sample were labeled using the Bioprime labeling kit from Invitrogen according to the manufacturer's instructions. The IP sample was labeled with Cy5-dUTP and the input DNA sample was labeled with Cy3-dUTP and $50 \mu 1$ of master mix(dNTPs-dATP, dGTP, dCTP; $120 \mu \mathrm{M}$, dTTP; $60 \mu \mathrm{M}$, Cy5-dUTP or Cy3-dUTP; $60 \mu \mathrm{M})$. After labeling the sample, the concentration was measured using an ND-1000 spectrometer (NanoDrop Technologies, Inc., Wilmington, DE).

Second, After checking labeling efficiency, each 2.5ug to 5ug of cyanine 3-labeled and cyanine 5-labeled DNA target were mixed and then resuspended with $2 \mathrm{X}$ hybridization buffer, Cot-1 DNA, and Agilent 10X blocking agent, and de-ionized formamide. Before hybridization to the array, the $260 \mathrm{ul}$ hybridization mixtures were denatured at $95^{\circ} \mathrm{C}$ for $3 \mathrm{~min}$ and incubated at $37^{\circ} \mathrm{C}$ for $30 \mathrm{~min}$. The hybridization mixtures were was centrifuged at $17,900 \mathrm{xg}$ for $1 \mathrm{~min}$ and directly pipetted onto the Customized Rat Methylation Microarray $(400 \mathrm{~K})$. The arrays hybridized at 65oC for $40 \mathrm{~h}$ using Agilent Hybridization oven (Agilent Technology, USA). The hybridized microarrays were washed as the manufacturer's washing protocol (Agilent Technology, USA). Third, after washing, hybridization images on the slides were scanned using the Agilent DNA microarray scanner (Agilent Technologies, USA) and signals were extracted from each probe using Agilent Feature Extraction software (v10.7.3.1). All data were normalized using Agilent's Workbench software v7.0 according to the manufacturer's instructions (Agilent Technologies, USA). The background-corrected intensity data were normalized with blank subtraction followed by intra-array LOWESS normalization. The peak detection was performed with Pre-defined Peak 
199 Shape detection v2.0 with a p-value $<0.01$ for non-parametric test and a peak-score $>5$ for 200 EVD-based score. The data were normalized by dividing the average of the signal intensity of 201 the exposed group by the normalized average of the control group. The differentially methylated 202 probes were selected using the 3.0-fold change cutoff and $p$-value $<0.05$. For reference, the 203 intensity dependent normalization is a technique that is used to eliminate dye-related artifacts in 204 two-color experiments that cause the cy5/cy3 ratio to be affected by the total intensity of the 205 spot. This normalization process attempts to correct for artifacts caused by non-linear rates of 206 dye incorporation as well as inconsistencies in the relative fluorescence intensity between some 207 red and green dyes.

208

209

210

211

\section{Integrating DNA methylation and gene expression}

To identify the anti-correlated methylated genes, we conducted a comparative analysis of DNA methylation and mRNA expression patterns using GeneSpring GX. mRNA profiles from the hexanal-exposed rats were obtained from our previous study (Cho et al., 2017). We used Pearson's correlation analysis, the most appropriate statistical coefficient for a small number of measures, to estimate the degree of anti-correlation (e.g., hyper methylation vs. down-regulated c mRNA expression or vice versa) between any putative pairs of DNA methylation and mRNA. The raw data are available from the NCBI GEO under accession number GSE60118. We considered the methylated genes with methylation differences of at least 3.0-fold and mRNA expression differences of at least 1.5 -fold on $p$-value $<0.05$.

\section{DAVID functional enrichment analysis}

Using the DAVID functional annotation bioinformatics tool, we performed GO enrichment analyses to understand biological functions associated with hexanal exposure. It was used to determine significant biological pathways for anti-correlated target genes between DNA methylation and mRNA expression associated with hexanal exposure. Fisher's exact test was used to detect significant enrichment of pathways, and the resulting p-value were adjusted using the Benjamini-Hochberg algorithm.

\section{Statistical analysis}

In all cases, the differences between the control and exposure group were evaluated using the unpaired t-test. The $p$-value criterion was set at $p$-value $<0.05$ as the level of statistical significance.

\section{Animal Ethics}

The experiment protocol was authorized by the Institutional Animal Care and Use Committee of 236

\section{Results}


238 Monitoring of inhalation exposure concentration, environmental conditions, and 239 histopathologic alterations

240 As mentioned in our previous studies (Cho et al., 2016; 2017), inhalation hexanal exposure

241 concentrations were monitored in rats using online gas chromatography (GC) every $10 \mathrm{~min}$

242 during the exposure period. SPF (Specific-pathogen-free) Fischer-344 derived (CRL:CD) rats of

243 both sexes were used at the age of 7 weeks ( $\mathrm{n}=10$ /group). The average exposure concentrations

244 were 646.03 ( \pm 80.06; low-dose), 999.06 ( \pm 162.08; middle-dose), and 1,525.31 ( \pm 199.02;

245 high- dose) $\mathrm{ppm}$. The conditions of the inhalation chamber such as temperature, relative

246 humidity, chamber pressure, and oxygen concentration were also measured (Cho et al., 2016).

247 Compared with the control group, no significant body weight, organ weight and histopathologic

248 alterations were observed after 4 weeks of hexanal exposure (Cho et al., 2017). In middle-dose

249 group, increased total bilirubin compared to control group in the male rats and decreased total

250 protein, albumin and triglyceride in the female rats were identified. These results showed no

251 significant dose-dependent changes related to hexanal exposure (Cho et al., 2017). Therefore, to

252 predict the potential adverse health effects of hexanal exposure we aimed to identify the hexnal-

253 associated genetic and epigenetic alterations using microarray-based mRNA and DNA

254

255

256 methylation to address the molecular basis of hexanal exposure relevant to respiratory system.

257

258

259

260

261

262

263

264

265

266

267

268

\section{DNA methylation pattern after hexanal exposure}

Aberrant DNA methylation has been linked to the abnormalities or disorders that induced by environmental stressors including environmental chemicals (Kubota, 2016). Therefore, the framework of epigenome for environmental risk assessment has been rapidly developed. First, we extracted from rats exposed to hexanal of three concentrations (Low dose, $600 \mathrm{ppm}$; Middle dose, 1,000 ppm; High dose, 1,500 ppm), and then genomic DNA using sonication to extract only methylated DNA using immunoprecipitation. The cleaved methylated DNA was confirmed using gel electrophoresis, and as a result, it was confirmed that the DNA of all groups was sheared to about $150 \mathrm{bp}$ to $500 \mathrm{bp}$, so that the optimal DNA for immunoprecipitation was secured (Supplementary Fig.1). After methylated DNA was extracted from aldehyde-exposed rat lung tissues through methylated DNA immunoprecipitation, the concentration was measured and the quantitative analysis of methylated DNA was performed through gel electrophoresis. As a result, it was confirmed that the concentration and state of methylated DNA are suitable for DNA methylation microarray (Supplementary Fig.2). In the current study, using a custom-designed

270 Agilent 400K CpG methylation microarray, we investigated DNA methylation profiles in $\mathrm{CpG}$ islands gene promoter sequences of hexanal-exposed lung tissues of F344 rats and compared

272 with those from rats exposed to clean filtered air (control group) (n=6/group). For reference, the DNA Methylation Microarray are designed to interrogate known $\mathrm{CpG}$ islands and related sites. It

275 is designed for analysis of methylated DNA derived from affinity-based isolation methods including methylated DNA immunoprecipitation (MeDIP). We analyzed methylation patterns for approximately 389,347 probes on the arrays. Compared with the control group, all three hexanal- 
278 signal that is acquired from normalizing the signal intensity by dividing the average of the signal 279 intensity of the control group. In the low dose exposure group, 661 methylated sites and 571 280 differentially methylated genes (hyper-methylated: 464, hypo-methylated: 107) were identified. 281 In the middle dose exposure group, 4,181 methylated sites and 3,268 differentially methylated

282

283

284

285

286

287

288

289

290

291

292

293

294

295

296

297

298

299

300

301

302

303

304

305

306

307

308

309

310

311

312

313

314

315

316 genes (hyper-methylated: 2,513, hypo-methylated: 755) were identified, and 11,744 methylated sites and 7,477 differentially methylated genes (hyper-methylated: 4,851, hypo-methylated:

2,662 ) were identified in the high dose exposure group. In all groups change was noted at $\geq 3.0$ fold change, $p$-value $<0.05$. Overall, the methylation sites increased as the exposure concentration increased. (Table 1).

Among these differentially methylated sites and genes, 79 sites and 73 genes (hyper-methylated: 69, hypo-methylated: 4) showed commonly methylated expression patterns in the three hexanal exposure groups (Fig. 2, Table 2). Furthermore, we identified 36 dose-dependent methylated genes (34 hyper-methylated and 2 hypo-methylated) in the common methylated genes of three hexanal exposure group using line-plot analysis (Fig. 3.A, Table 3). The dose-dependent genes are illustrated as a heatmap (Fig. 3.B). These dose-response relationships have the potential to serve as quantitative epigenetic biomarkers of hexanal exposure. Raw data are available online at Gene Expression Omnibus (GEO accession number GSE129313).

\section{Gene expression profiles induced by hexanal exposure}

To investigate the gene expression signatures response to hexanal inhalation exposure, we previously investigated the gene expression profiling of lung tissues of hexanal-exposed F344 rats using the Rat Oligo Microarray $(44 \mathrm{~K})$. The raw data are available at GEO/NCBI GSE 60118. The gene expression profiles were analyzed by comparing them to the control group using 1.5 -fold change and unpaired t-test $p$ value $<0.05$ as statistical significance (Table 4 ). In the previous study, we identified hexanal specific genes that were involved in diverse biological processes including apoptosis, cell proliferation, and mitogen-activated protein kinase (MAPK) cascade. These genes were also associated with disease such as respiratory and nervous system diseases (Cho et al., 2017). It suggests that hexanal exposure may have potential adverse health effects on humans. Therefore, we aimed to analyze DNA methylation signatures in hexanalexposed F344 rats to understand the epigenetic effects of hexanal exposure.

\section{Comparative analysis of DNA methylation and mRNA expression profiles}

DNA methylation was involved in transcriptional regulation and gene activity. Promoter hypermethylation can leads to silencing of gene expression, whereas hypo-methylation can leads to gene activation. The investigation of the implication of DNA methylation in the regulation of gene expression and identification of key genes that regulated by both DNA methylation and gene expression using integrative analysis is important. Therefore, we conducted an integrated analysis of DNA methylation (Table 1.) and mRNA expression data (Table 4.). As shown in Table 5, we identified the hyper-methylated vs. down-regulated genes and hypo-methylated vs. 
317 up-regulated genes in the hexanal exposure groups. These results suggest potential core DNA

318

319

320

321

322

323

324

325

326

327

328

329

330

331

332

333

334

335

336

337

338

339

340

341

342

343

344

345

346

347

348

349

350

351

352

353

354

355

356

methylation-based epigenetic biomarkers for exposure/risk assessment of hexanal.

\section{Gene Ontology (GO) analysis of putative DNA methylation biomarkers of hexanal}

We next investigated the relevant molecular and cellular processes controlled by hexanal exposure-specific inversely correlated target genes based on GO biological processes terms using the DAVID bioinformatics tool (Table 6). The key GO terms were related to the lactation (GO: 0007595), skeletal muscle cell differentiation (GO:0035914), Positive regulation of synapse assembly (GO:0051965), sodium ion transport (GO:0006814), and regulation of tumor necrosis factor production (GO:0032680. These results indicated that putative epigenetic biomarkers of hexanal are involved in skeletal muscle cell differentiation, synapse assembly, and TNF production. Further studies are necessary to determine hexanal-induced toxicological mechanisms based on functional enrichment analysis.

\section{Discussion}

Traditional toxicity testing depends on animal testing to investigate the risk of chemicals to human health. It requires several animals, high investment, and a significant amount of time. Additionally, it should consider ethical treatment of animals and their welfare. Therefore, this approach is insufficient to handle risk assessments of the large number of chemicals in the environment (Chen et al., 2012; North \& Vulpe, 2010), and novel strategies for toxicological risk assessment of environmental chemicals are necessary.

In response to these challenges, the field of toxicogenomics has been established and developed rapidly for risk assessments. Toxicogenomics includes high-throughput technologies such as transcriptomics, proteomics, and metabolomics for predictive toxicology and risk assessment (Hamadeh et al., 2002). Currently, an integrated framework for multi-omics has been proposed.

It provides insight into the mode of action of environmental toxicants and helps in understanding the underlying mechanisms of toxicants and adverse outcome pathways (AOPs) (Williams et al., 2014). In contrast to traditional toxicity methods, it is possible to also identify multiple-response and endpoints using toxicogenomics.

Toxicogenomics study has developed rapidly with microarray and next generation sequencing technologies. The microarray technology was proposed in the 1990s (Chen et al., 2012). It is a powerful tool for evaluating the effect of environmental chemicals on human health, providing valuable genomic information for identifying biomarkers related to occupational exposure and disease prognosis (Jung et al., 2017; Gwinn \& Weston, 2008; Kim et al., 2016). It allows simultaneous screening of the expression levels of thousands of genes exposed to environmental toxicants based on omics tools. Therefore, toxicogenomics has been considered as a new toxicology paradigm for risk assessment and prediction of exposure and risk of environmental chemicals.

One of the epigenome studies demonstrated that DNA methylation has an important role in the regulation of gene expression and epigenetic phenotype variation (Hong et al., 2018) leading to 
357

358

359

360

361

362

363

364

365

366

367

368

369

370

371

372

373

374

375

376

377

378

379

380

381

382

383

384

385

386

387

388

389

390

391

392

393

394

395

396

insights into the development of diseases associated with environmental risk assessment (Ray et al., 2014; Conerly \& Grady; 2010). Generally, the expression patterns of DNA methylation are altered by environmental factors, including environmental chemicals, air pollution, and nonchemical stressors. Moreover, it has been linked to levels of health, disease susceptibility, and disease development (Martin \& Fry; 2018). Therefore, epigenetic modifications can be novel exposure biomarkers of the diseases related to environmental factors.

To investigate the epigenetics actions of hexanal associated with lung toxicity, we aimed to identify epigenetic biomarkers based on DNA methylation. As major component of indoor air pollutants, we previously analyzed the transcriptome profiles of hexanal using in vitro and in vivo models (Cho et al., 2014; 2017). And we also analyzed the methylation profiles of seven aldehydes (propanal, butanal, pentanal, hexanal, heptanal, octanal, and nonanal) exposed human lung epithelial cell, A549, to investigate the aldehydes exposure and epigenetic alterations based on DNA methylation (Cho et al., 2018). Here, we proposed three steps of DNA methylome analysis of hexanal exposure using the in vivo model. First, we identified the differentially methylated genes of hexanal exposure showing a 3.0-fold-change $(p<0.05)$. Of the 389,347 probes on the customized rat $400 \mathrm{~K} \mathrm{CpG}$ methylation microarray, the methylated genes identified showed significant expression changes in the three hexanal exposure groups (low dose, middle dose, and high dose) compared to the control group. Among the differentially methylated genes, we identified commonly methylated genes and dose-dependent methylated patterns, which provided significant novel epigenetic biomarkers of hexanal exposure. These methylated genes were involved in chemical stimulus associated with olfactory receptor activity (OLR1696, OLR500, OLR5, OLR407, OLR1085, OLR1084, OLR1389), insulin stimulus (PLA2G1B, MYO5A, USF1) and negative regulation of peptidyl-serine phosphorylation (HGF, INPP5J). The follow-up studies will be necessary to address a pulmonary toxicological mechanisms associated with hexanal exposure. Also, the dose-response relationship plays essential role in the field of toxicology, it provides the determination of threshold for toxic effect and better understanding of network for exposure-human health (Tsatsakis et al., 2018).

Second, we analyzed the transcriptome profiles of hexanal exposure in F344 rats to investigate the hexanal-characterized genes and environmental chemical-gene interactions based on toxicogenomics (Cho et al., 2017). Third, we conducted the comparative analysis of genomewide DNA methylome and transcriptome in the hexanal- exposed F344 rats. It is well known that the DNA methylation is associated with gene expression. DNA hypermethylation results in gene silencing and hypomethylation leads to elevated transcription (Li et al., 2017). The identification of key genes that regulated by both DNA methylation and mRNA expression system via integrative analysis is necessary. Therefore, we aimed to identify the novel biomarkers that anticorrelated between DNA methylation and gene expression. Together, these processes can serve to determine the important framework for environmental epigenetics in exposure/risk assessment and it allows the identification of the critical bridging epigenetic biomarkers of hexanal. Further biomarker validation and developing studies are necessary to explore the specificity, sensitivity and implications of these biomarkers. And then, it is predicted that this epigenetic biomarkers 
397 398

399

400

401

402

403

404

405

406

407

408

409

410

411

412

413

414

415

416

417

418

419

420

421

422

423

424

425

426

427

428

429

430

431

432

433

434

435

436

can be used to determine whether exposure to hexanal and to determine the cause of environmental diseases.

In vivo models including rat model are essential for evaluating the toxicity of inhaled factors for the risk assessment on human health. It is fundamental for understanding the mammalian system including human biology at molecular level. Therefore, we used the F344 rat models to evaluate the pulmonary toxicity of hexanal associated with human adverse health effects. In this study, the analyzed DNA methylated genes at $\mathrm{CpG}$ islands were conserved in human. It has orthologs between rat and human.

Most of aldehydes inhalation toxicity research has progressed extensively on formaldehyde and acetaldehyde, which are classified as Group 1 carcinogenes by IARC (International Agency for Research on Cancer). However, other aldehydes such as hexanal toxicological data are relatively insufficient for risk assessment. Therefore, we aimed to investigate the inhalation toxicity of hexanal using F344 rats. For reference, in this study, hexanal exposure doses (low dose,600 ppm; middle dose, 1,000 ppm; and high dose, 1,500 ppm) were selected based on the $\mathrm{LC}_{\mathrm{Lo}}$ (Lowest Lethal Concentration; 2,000 ppm/4hr) of hexanal using nose-only inhalation chamber. These exposure dose levels that are much higher than actually exposed levels in environment. Since the VOCs are typically exposed to low levels for long-term, we determined the hexanal exposure doses higher than the actual exposure levels to investigate the clear implications for human health.

Using the DAVID functional annotation bioinformatics tool, GO analysis was also performed. GO enrichment analysis demonstrated that cell differentiation of skeletal muscle cells, regulation of synapse assembly and regulation of TNF production are involved in major biological process associated with hexanal exposure. Among them, BTG2, ZFP36 and ASIC2 were commonly involved in hexanal related biological processes such as skeletal muscle cell differentiation and regulation of nuclear-transcribed mRNA poly (A) tail shortening. BTG2 (BTG anti-proliferation factor 2) has important roles in control of cell growth, cell differentiation, apoptosis and transcriptional regulation. Moreover, it is involved in tumor progression in response to a variety of stressors, steroid hormones and growth factors (Yuniati L et al., 2019). ZFP36 (Zinc finger protein 36 homolog; also known as Tristetraprolin) plays role in regulation of TNF- $\alpha$ (Tumor necrosis factor-alpha) expression which is a pro-inflammatory mediator (Zhao XK et al., 2016). Since we identified that the relationship between the TNF regulation and hexanal exposure using GO analysis, we considered that further research on inflammatory mechanisms via TNF associated with ZFP36 expression is required. ASIC2 (Acid sensing ion channel subunit 2) is expressed in several systems including peripheral and central nervous system as mechanoreceptor and acid receptor (Kikuchi et al., 2008). Recent studies demonstrated that ASIC2 may lead to increase the pulmonary vascular resistance and possibility of hypoxic pulmonary hypertension (Detweiler et al., 2019).

These results reflect that hexanal exposure may affect skeletal muscle and neuronal system as well as respiratory system. Further validation of key toxicological mechanisms induced by hexanal exposure such as pulmonary inflammation via TNF signaling pathway is required. 


\section{Conclusions}

439

440

441

442

443

444

445

446

447

448

449

450

451

452

453

454

455

456

457

458

459

460

461

462

463

464

465

466

467

468

469

470

471

472

473

474

475

476

Taken together, this study demonstrated the characteristic methylated profiles by hexanal inhalation exposure system using DNA methylome analysis in an in vivo model. By integrating DNA methylation and mRNA expression profiles, target genes were identified. These genes could be valuable epigenetic biomarkers to distinguish exposure to hexanal and to determine the DNA methylome responses to hexanal exposure in the environment and to predict the underlying mechanisms of hexanal exposure associated with pulmonary toxicity. Further studies on these methylated signatures are required to provide insights into the molecular toxicological mechanisms activated by hexanal exposure.

\section{Acknowledgements}

\section{Conflict of Interest}

The authors declare that there are no conflicts of interest.

\section{Author Contributions}

Yoon Cho performed the experiments, analyzed the data, and wrote the manuscript; Mi-Kyung

Song designed the research and performed the experiments; Jae-Chun Ryu conceived and designed the research, and supervised the work.

\section{References}

Baccarelli, A., Bollati, V. Epigenetics and environmental chemicals. Curr Opin Pediatr. 21(2), 243-251 (2009).

Burris, H.H., Baccarelli, A.A. Environmental epigenetics: from novelty to scientific discipline. J Appl Toxicol. 34(2), 113-116 (2014).

Chen, M., Zhang, M., Borlak, J., Tong, W. A decade of toxicogenomic research and its contribution to toxicological science. Toxicol Sci. 130(2), 217-228 (2012).

Cho, Y., Song, M.K., Choi, H.S., Ryu, J.C. Analysis of Dose-response to Hexanal-induced Gene Expression in A549 Human Alveolar Cells. BioChip J. 8(2), 75-82 (2014).

Cho, Y, Lim J.H., Jeong, S.C., Song, M.K., Ryu J.C. Hexanal-induced Changes in miRNAmRNA Interactions in A549 Human Alveolar Epithelial Cells. Toxicol. Environ. Health. Sci. 7(2), 143-159 (2015).

Cho, Y., Song, M.K., Jeong, S.C., Lee, K., Heo, Y., Kim, T.S., Ryu, J.C. MicroRNA response of inhalation exposure to hexanal in lung tissues from Fischer 344 rats. Environ Toxicol. 31(12), 1909-1921 (2016).

Cho, Y., Lim, J.H., Song, M.K., Jeong, S.C., Lee, K., Heo, Y., Kim, T.S., Ryu, J.C.

Toxicogenomic analysis of the pulmonary toxic effects of hexanal in F344 rat. Environ Toxicol. 32(2), 382-396 (2017).

Cho, Y., Song, M.K., Kim, T.S., Ryu, J.C. DNA Methylome Analysis of Saturated Aliphatic Aldehydes in Pulmonary Toxicity. Sci Rep. 8(1):10497 (2018). 
477 Conerly, M., Grady, W.M. Insights into the role of DNA methylation in disease through the use

478 of mouse models. Dis Model Mech. 3(5-6), 290-297 (2010).

479 Detweiler ND, Herbert LM, Garcia SM, Yan S, Vigil KG, Sheak JR, Resta TC, Walker BR, 480 Jernigan NL. Loss of acid-sensing ion channel 2 enhances pulmonary vascular resistance and 481 hypoxic pulmonary hypertension. J Appl Physiol (1985). 127(2), 393-407 (2019).

482 Garcia-Alcega, S., Nasir, Z.A., Ferguson, R., Noël, C., Cravo-Laureau, C., Whitby, C., 483 Dumbrell, A.J., Colbeck, I., Tyrrel, S., Coulon, F. Can chemical and molecular biomarkers help 484 discriminate between industrial, rural and urban environments?Science of The Total 485 Environment. 631-632, 1059-1069 (2018).

486 Gwinn, M.R., Weston, A. Application of oligonucleotide microarray technology to toxic 487 occupational exposures. J Toxicol Environ Health A. 71(5), 315-324 (2008).

488 Hamadeh, H.K., Amin, R.P., Paules, R.S., Afshari, C.A. An overview of toxicogenomics. Curr 489 Issues Mol Biol. 4(2), 45-56 (2002).

490 Ho, S.M., Johnson, A., Tarapore, P., Janakiram, V., Zhang, X., Leung, Y.K. Environmental 491 epigenetics and its implication on disease risk and health outcomes. ILAR J. 53(3-4), 289-305 492 (2012).

493 Hong, Y., Hong, S.-H., Oh, Y.-M., Shin, S.-H., Choi, S.S., Kim, W.J. Identification of lung 494 cancer specific differentially methylated regions using genome-wide DNA methylation study.

495 Mol Cell Toxicol. 14, 315-322 (2018).

496 Jung, J., Hah, K., Lee, W., Jang, W. Meta-analysis of microarray datasets for the risk assessment 497 of coplanar polychlorinated biphenyl 77 (PCB77) on human health. Toxicol. Environ. Health. 498 Sci. 9(2), 161-168 (2017).

499 Kagohara, L.T., Stein-O'Brien, G.L., Kelley, D., Flam, E., Wick, H.C., Danilova, L.V., 500 Easwaran, H., Favorov, A.V., Qian, J., Gaykalova, D.A., Fertig, E.J. Epigenetic regulation of 501 gene expression in cancer: techniques, resources and analysis. 17(1), 49-63 (2018).

502 Kikuchi S, Ninomiya T, Kawamata T, Tatsumi H. Expression of ASIC2 in ciliated cells and 503 stereociliated cells. Cell Tissue Res. 333(2), 217-24 (2008).

504 Kim, S.Y., Hong, J.Y., Yu, S.Y., Kim, G.W., Ahn, J.J., Kim, Y., Son, S.W., Park, J.T., Hwang, 505 S.Y. Identification of potential biomarkers for xylene exposure by microarray analyses of gene 506 expression and methylation. Mol. Cell. Toxicol. 12(1), 15-20 (2016).

507 Koh, E.J., Hwang, S.Y. Multi-omics approaches for understanding environmental exposure and 508 human health. Mol Cell Toxicol. 15, 1-7 (2019).

509 Korpi, A., Järnberg, J., Pasanen, A.L. Microbial volatile organic compounds. Crit Rev Toxicol. 510 39(2), 139-193 (2009).

511 Kubota, T. Epigenetic alterations induced by environmental stress associated with metabolic and 512 neurodevelopmental disorders. Environ Epigenet.2(3):dw017 (2016).

513 Li, Z., Zhuang, X., Zeng, J., Tzeng, C.M. Integrated Analysis of DNA Methylation and mRNA 514 Expression Profiles to Identify Key Genes in Severe Oligozoospermia. Front Physiol. 8:261 515 (2017). 
516 Martin, E.M., Fry, R.C. Environmental Influences on the Epigenome: Exposure- Associated 517 DNA Methylation in Human Populations. Annu Rev Public Health. 39:309-333 (2018).

518 McHale, C.M., Zhang, L., Hubbard, A.E., Smith, M.T. Toxicogenomic profiling of chemically 519 exposed humans in risk assessment. Mutat Res. 705(3), 172-183 (2010).

520 North, M., Vulpe, C.D. Functional toxicogenomics: mechanism-centered toxicology. Int J Mol 521 Sci. 11(12), 4796-4813 (2010).

522 Szyf, M. The implications of DNA methylation for toxicology: toward toxicomethylomics, the 523 toxicology of DNA methylation. Toxicol Sci. 120(2): 235-255 (2011).

524 Thorn, R.M., Greenman, J. Microbial volatile compounds in health and disease conditions. J

525 Breath Res. 6(2), 024001 (2012).

526 Tsatsakis, A.M., Vassilopoulou, L., Kovatsi, L., Tsitsimpikou, C., Karamanou, M., Leon, G.,

527 Liesivuori, J., Hayes, A.W., Spandidos, D.A. The dose response principle from philosophy to

528 modern toxicology: The impact of ancient philosophy and medicine in modern toxicology

529 science. Toxicol Rep. 5:1107-1113 (2018).

530 Ray, P.D., Yosim, A., Fry, R.C. Incorporating epigenetic data into the risk assessment process

531 for the toxic metals arsenic, cadmium, chromium, lead, and mercury: strategies and challenges.

532 Front Genet. 5, 201. doi: 10.3389/fgene.2014.00201 (2014).

533 Reamon-Buettner, S.M., Mutschler, V., Borlak, J. The next innovation cycle in toxicogenomics:

534 environmental epigenetics. Mutat Res. 659(1-2), 158-65 (2008).

535 Watson, R.E., Goodman, J.I. Epigenetics and DNA methylation come of age in toxicology.

536 Toxicol Sci. 67(1), 11-16 (2002).

537 Williams, T.D., Mirbahai, L., Chipman, J.K. The toxicological application of transcriptomics and

538 epigenomics in zebrafish and other teleosts. Brief Funct Genomics. 13(2), 157-171 (2014).

539 Yuniati L, Scheijen B, van der Meer LT, van Leeuwen FN. Tumor suppressors BTG1 and

540 BTG2: Beyond growth control. J Cell Physiol. 234(5), 5379-5389 (2019).

541 Zhao XK, Che P, Cheng ML, Zhang Q, Mu M, Li H, Luo Y, Liang YD, Luo XH, Gao CQ,

542 Jackson PL, Wells JM, Zhou Y, Hu M, Cai G, Thannickal VJ, Steele C, Blalock JE, Han X,

543 Chen CY, Ding Q. Tristetraprolin Down-Regulation Contributes to Persistent TNF-Alpha

544 Expression Induced by Cigarette Smoke Extract through a Post-Transcriptional Mechanism.

545 PLoS One. 11(12), e0167451. doi: 10.1371/journal.pone.0167451 (2016).

546 Zoghbi, H.Y., Beaudet, A.L. Epigenetics and Human Disease. Cold Spring Harb Perspect Biol.

$5478(2)$, a019497 (2016).

548 


\section{Figure 1}

Total DNA methylation expression profiles of hexanal exposed in F-344 rats ( $n=6 /$ group).

Total DNA methylation expression profiles of hexanal exposed in F-344 rats ( $n=6 /$ group). Two-dimensional diagram of the characteristic expression profiles of 389,247 classifier methylation probes. Rows (y-axis) represent the intensity of the DNA methylation probes and columns (x-axis) represent the different experimental conditions. Color intensity reflects differences in expression between sample DNA and reference DNA.

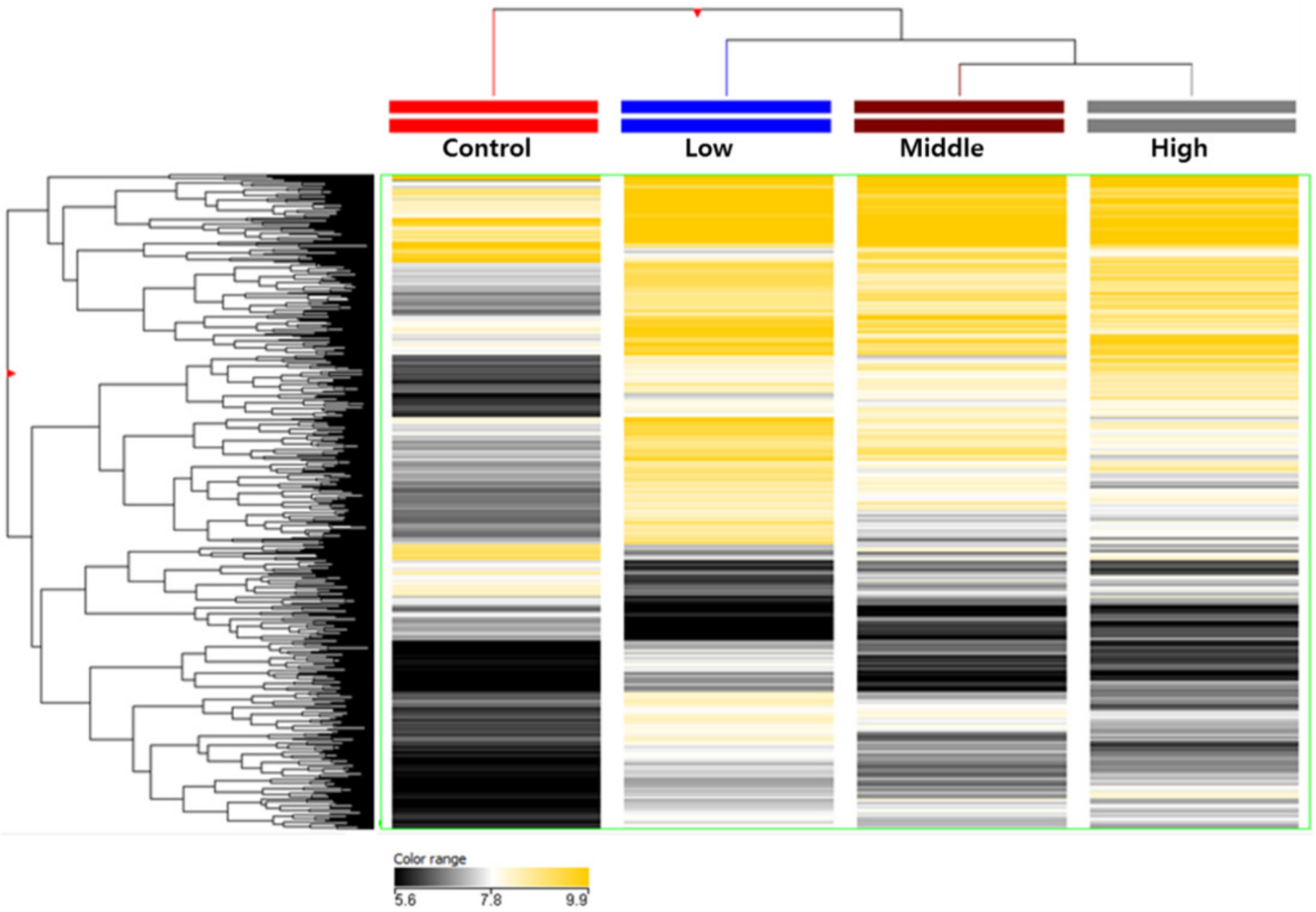


Figure 2

The Venn diagram and hierarchical clustering image of hexanal specific methylated DNA

(A) The Venn diagram and (B) hierarchical clustering image shows that 73 methylated DNA that commonly altered their expression are identified in three dose - T1(600ppm), T2 (1,000ppm) and T3(1,500ppm) with a fold-change $\geq 3.0$-fold and $p$-value $<0.05$ compared to the control group (Filtered air) (Yellow: hypermethylation; Black: hypomethylation).

A

Low dose

(T1)

Middle dose

(T2)

174

20

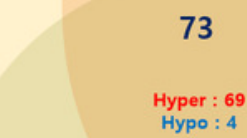

304

Hyper : 69

2,217

4,883

High dose

(T3)

B

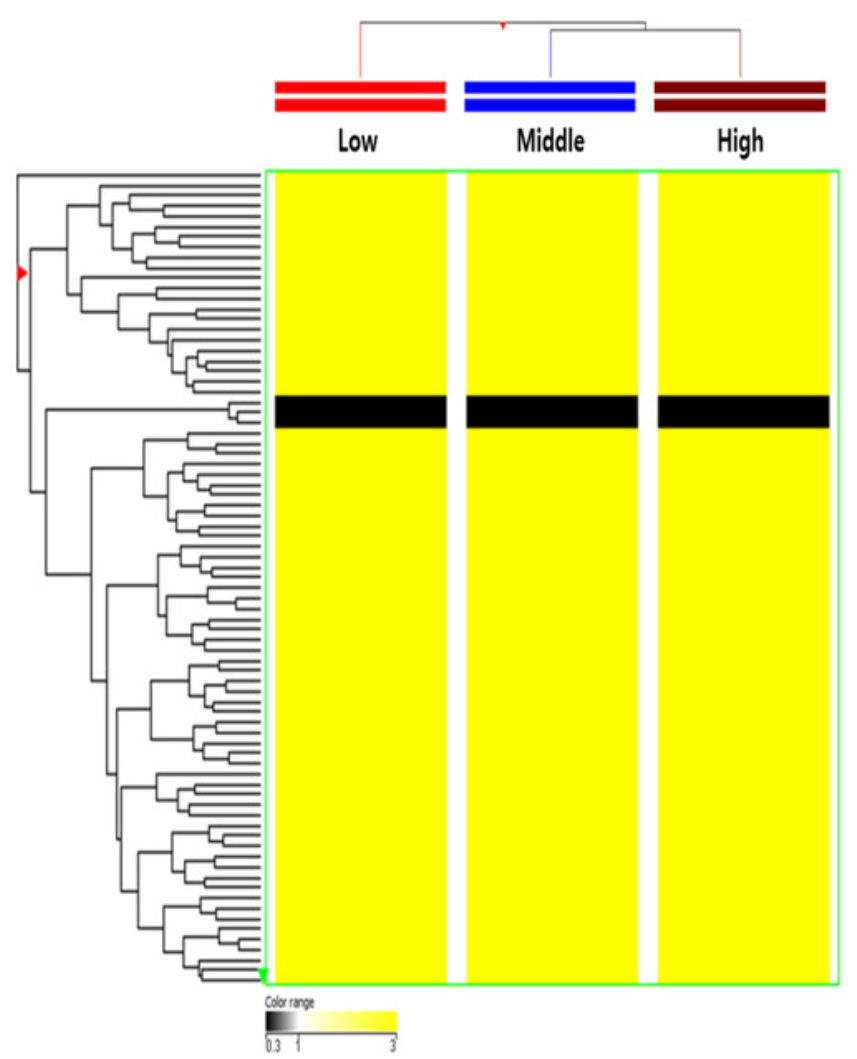


Figure 3

Line plot and heatmap of dose-dependent response of methylated genes by hexanal exposure

(A) Line plot showed dose-dependent response of methylated genes by hexanal exposure. Each line of the plot represents the normalized intensity values by the control group shown on the $x$-axis. The $y$-axis has a log2 scale. (B) The heatmap of 36 dose-dependent methylated genes by hexanal exposure.

A

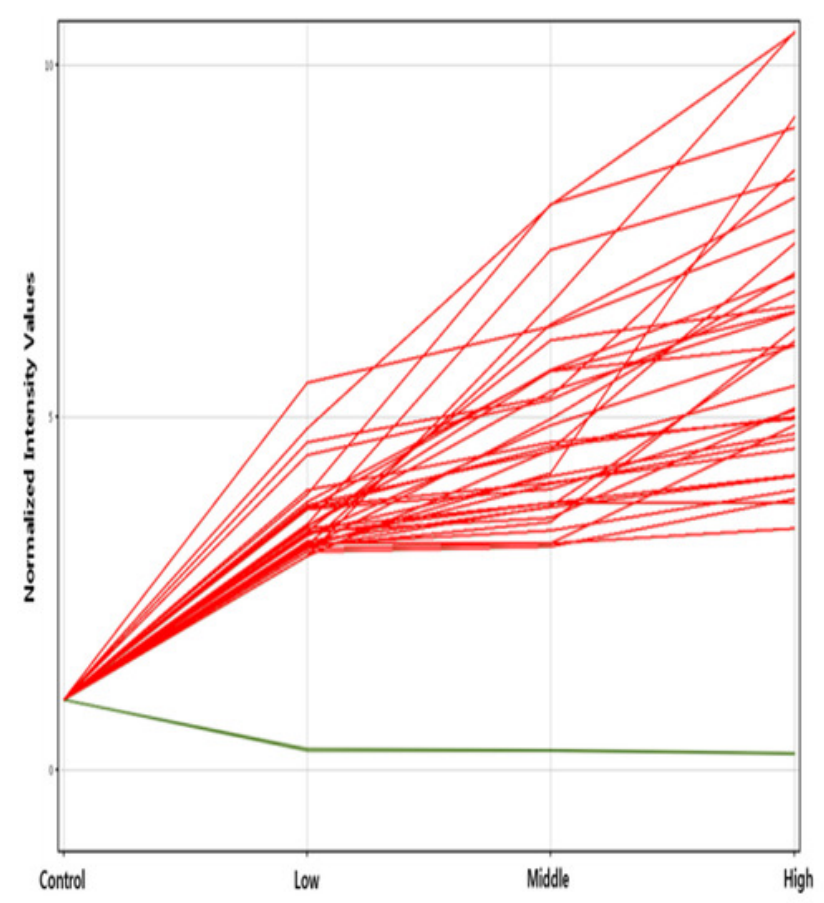

B

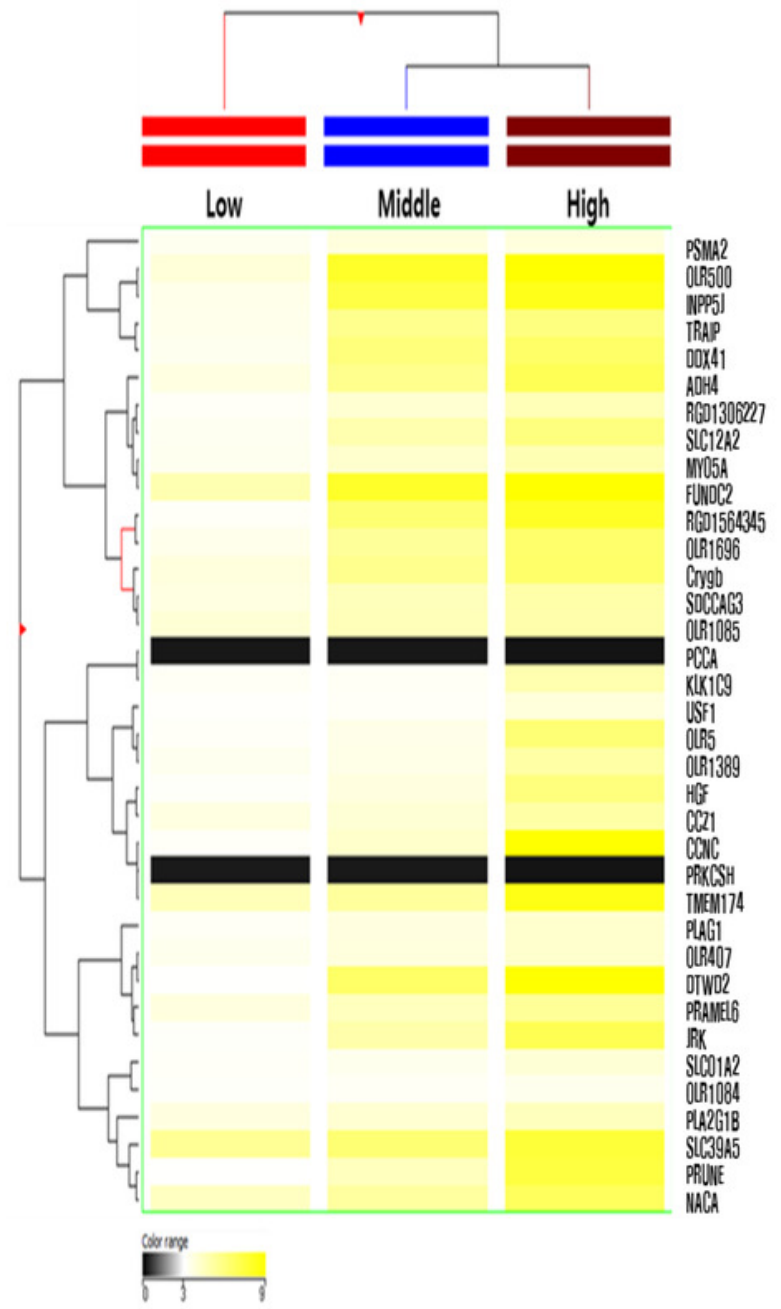




\section{Table $\mathbf{1}$ (on next page)}

The DNA methylated sites and regulated target genesin three hexanal exposure group. 
1 Table 1. The DNA methylated sites and regulated target genes in three hexanal exposure group.

\begin{tabular}{ccc}
\hline Exposure dose & Methylated sites & Regulated target genes \\
\hline Low dose $(600 \mathrm{ppm})$ & 661 & 571 \\
Middle dose $(1,000 \mathrm{ppm})$ & 4,181 & 3,268 \\
High dose $(1,500 \mathrm{ppm})$ & 11,744 & 7,477 \\
\hline
\end{tabular}

2 


\section{Table 2 (on next page)}

The list of 73 methylated target genes thatcommonly altered their expression in three hexanal exposure group.

The list of 73 methylated target genes that commonly altered their expression in three hexanal exposure group. 
1 Table 2. The list of 73 methylated target genes that commonly altered their expression in three hexanal exposure group.

\begin{tabular}{|c|c|c|c|c|c|}
\hline \multirow[b]{2}{*}{ Probe ID } & \multirow[b]{2}{*}{ Annotation } & \multicolumn{3}{|c|}{ Fold change } & \multirow[b]{2}{*}{ Regulation } \\
\hline & & $\begin{array}{l}\text { Low dose } \\
\text { (T1) }\end{array}$ & $\begin{array}{l}\text { Middle dose } \\
\text { (T2) }\end{array}$ & $\begin{array}{l}\text { High dose } \\
\text { (T3) }\end{array}$ & \\
\hline RP14104253 & Keng1 & 0.31 & 0.27 & 0.33 & down \\
\hline RP14316148 & Pcca & 0.28 & 0.28 & 0.23 & down \\
\hline RP14196310 & Prkcsh & 0.30 & 0.29 & 0.24 & down \\
\hline RP14232470 & Sstr5 & 0.27 & 0.29 & 0.18 & down \\
\hline RP14072666 & Adh4 & 3.69 & 5.67 & 6.99 & up \\
\hline RP14052111 & Ankrd34b & 3.34 & 5.67 & 3.86 & up \\
\hline RP14347714 & Atp9b & 3.82 & 5.05 & 3.70 & up \\
\hline RP14104488 & Bcas1 & 4.10 & 3.07 & 6.10 & up \\
\hline RP14068918 & Capza1 & 4.21 & 3.73 & 5.01 & up \\
\hline RP14275323 & $\mathrm{Ccl} 24$ & 3.38 & 3.97 & 3.64 & up \\
\hline RP14132603 & Ccnc & 3.17 & 4.21 & 9.26 & up \\
\hline RP14271990 & Cczl & 3.69 & 3.98 & 5.10 & up \\
\hline RP14133935 & Chmp5 & 3.55 & 3.30 & 3.65 & up \\
\hline RP14222274 & Crygb & 3.80 & 5.65 & 6.50 & up \\
\hline RP14271525 & Сyp3a23/3a1 & 3.04 & 4.79 & 4.29 & up \\
\hline RP14329375 & $\mathrm{Ddx} 41$ & 3.38 & 6.09 & 6.58 & up \\
\hline RP14200524 & Dpagt1 & 3.15 & 3.10 & 4.53 & up \\
\hline RP14344056 & Dtwd2 & 3.03 & 6.60 & 10.47 & up \\
\hline RP14214176 & Eiflb & 4.11 & 4.07 & 12.6 .37 & up \\
\hline RP14372792 & Fam228a & 3.13 & 4.24 & 3.59 & up \\
\hline RP14266770 & Fgf12 & 4.25 & 3.27 & 3.56 & up \\
\hline RP14338669 & Fundc2 & 4.84 & 8.01 & 10.45 & up \\
\hline RP14253846 & G6pc3 & 4.79 & 3.86 & 3.74 & up \\
\hline
\end{tabular}




\begin{tabular}{llllll} 
RP14008399 & Gltscr2 & 3.38 & 4.32 & 4.30 & up \\
\hline RP14108468 & Hgf & 3.15 & 3.74 & 6.07 & up \\
\hline RP14139108 & Hook1 & 4.25 & 3.50 & 5.47 & up \\
\hline RP14301839 & Inpp5j & 3.49 & 7.37 & 8.39 & up \\
\hline RP14183524 & Jrk & 3.15 & 4.97 & 7.04 & up \\
\hline RP14214724 & Kif15 & 3.23 & 4.71 & 4.62 & up \\
\hline RP14016091 & K1k1c9 & 3.21 & 3.21 & 4.89 & up \\
\hline RP14248629 & LOC303448 & 3.74 & 5.28 & 4.63 & up \\
\hline RP14343955 & LOC317165 & 3.44 & 4.13 & 3.41 & up \\
\hline RP14299892 & Lyar & 3.51 & 4.27 & 3.36 & up \\
\hline RP14208423 & Mrap2 & 3.18 & 5.16 & 4.73 & up \\
\hline RP14207074 & My05a & 3.37 & 4.16 & 4.77 & up \\
\hline RP14169885 & Naca & 4.46 & 5.24 & 6.78 & up \\
\hline RP14301172 & Nelfa & 4.68 & 3.28 & 4.22 & up \\
\hline RP14171345 & Olr1049 & 3.31 & 3.21 & 4.30 & up \\
\hline RP14175180 & Olr1084 & 3.14 & 3.22 & 3.42 & up \\
\hline RP14175183 & Olr1085 & 3.97 & 4.65 & 4.98 & up \\
\hline RP14199556 & Olr1328 & 3.99 & 3.65 & 5.69 & up \\
\hline RP14235002 & Olr1389 & 3.38 & 3.57 & 5.12 & up \\
\hline RP14307926 & Olr1624 & 9.62 & 24.83 & 12.97 & up \\
\hline RP14359583 & Olr1696 & 3.46 & 5.37 & 6.49 & up \\
\hline RP14359635 & Olr1701 & 4.19 & 3.05 & 5.11 & up \\
\hline RP14080399 & Olr407 & 3.43 & 3.79 & 4.18 & up \\
\hline RP14085578 & Olr448 & 3.37 & 4.72 & 4.11 & up \\
\hline RP14006401 & Olr5 & 3.17 & 3.51 & 6.25 & up \\
\hline RP14086255 & Olr500 & 3.90 & 8.02 & 9.10 & up \\
\hline RP14342389 & Pcdhb12 & 3.26 & 3.18 & 3.44 & up \\
\hline & & & & \\
\hline
\end{tabular}




\begin{tabular}{llllll} 
RP14280875 & Pitpnb & 3.81 & 5.59 & 4.57 & up \\
\hline RP14279738 & Pla2g1b & 3.73 & 4.07 & 4.56 & up \\
\hline RP14131422 & Plag1 & 3.24 & 3.72 & 4.16 & up \\
\hline RP14086989 & Prame16 & 3.75 & 4.52 & 5.44 & up \\
\hline RP14066541 & Prune & 3.03 & 4.54 & 7.46 & up \\
\hline RP14335189 & Psma2 & 3.35 & 3.78 & 3.78 & up \\
\hline RP14055154 & RGD1306227 & 3.18 & 4.06 & 4.69 & up \\
\hline RP14108982 & RGD1564345 & 3.18 & 6.32 & 8.11 & up \\
\hline RP14374247 & Rhox3 & 3.24 & 4.87 & 3.71 & up \\
\hline RP14029004 & rnf141 & 3.05 & 8.04 & 5.87 & up \\
\hline RP14212856 & Rtp3 & 3.35 & 6.35 & 5.19 & up \\
\hline RP14076254 & Sdccag3 & 3.69 & 4.58 & 5.01 & up \\
\hline RP14344660 & Slc12a2 & 3.32 & 4.88 & 6.02 & up \\
\hline RP14170272 & Slc39a5 & 5.49 & 6.28 & 7.65 & up \\
\hline RP14130014 & Slco1a2 & 3.20 & 3.40 & 3.96 & up \\
\hline RP14165691 & Slirp & 3.82 & 4.60 & 4.47 & up \\
\hline RP14049010 & Taf5 & 3.19 & 4.32 & 3.27 & up \\
\hline RP14053110 & Tmem174 & 5.15 & 4.73 & 4.69 & up \\
\hline RP14211530 & Traip & 3.45 & 5.65 & 6.02 & up \\
\hline RP14096780 & Trmt6 & 4.06 & 3.93 & 5.93 & up \\
\hline RP14013877 & Tyrobp & 3.60 & 4.79 & 3.66 & up \\
\hline RP14288987 & Usf1 & 3.09 & 3.16 & 3.85 & up \\
\hline RP14237317 & Zfp672 & 3.69 & 5.22 & 4.65 & up \\
\hline & & & & \\
\hline
\end{tabular}




\section{Table 3(on next page)}

The dose-dependent methylated target genesin three hexanal exposure group. 
1 Table 3. The dose-dependent methylated target genes in three hexanal exposure group.

\begin{tabular}{|c|c|c|c|c|c|}
\hline \multirow{2}{*}{ Probe ID } & \multirow{2}{*}{ Annotation } & \multicolumn{3}{|c|}{ Fold change } & \multirow{2}{*}{ Regulation } \\
\hline & & $\begin{array}{l}\text { Low dose } \\
(600 \text { ppm) }\end{array}$ & $\begin{array}{l}\text { Middle dose } \\
(1,000 \text { ppm) }\end{array}$ & $\begin{array}{c}\text { High dose } \\
(1,500 \text { ppm })\end{array}$ & \\
\hline RP14196310 & Prkcsh & 0.30 & 0.29 & 0.24 & Down \\
\hline RP14316148 & Pcca & 0.28 & 0.28 & 0.23 & Down \\
\hline RP14006401 & Olr5 & 3.17 & 3.51 & 6.25 & Up \\
\hline RP14016091 & Klk1c9 & 3.21 & 3.21 & 4.89 & Up \\
\hline RP14053108 & Tmem174 & 4.64 & 5.29 & 8.50 & Up \\
\hline RP14055154 & RGD1306227 & 3.18 & 4.06 & 4.69 & Up \\
\hline RP14066541 & Prune & 3.03 & 4.54 & 7.46 & Up \\
\hline RP14072666 & Adh4 & 3.69 & 5.67 & 6.99 & Up \\
\hline RP14076254 & Sdccag3 & 3.69 & 4.58 & 5.01 & $\mathrm{Up}$ \\
\hline RP14080399 & Olr407 & 3.43 & 3.79 & 4.18 & Up \\
\hline RP14086255 & Olr500 & 3.90 & 8.02 & 9.10 & $\mathrm{Up}$ \\
\hline RP14086989 & Pramel6 & 3.75 & 4.52 & 5.44 & Up \\
\hline RP14108468 & $\operatorname{Hgf}$ & 3.15 & 3.74 & 6.07 & Up \\
\hline RP14108982 & RGD1564345 & 3.18 & 6.32 & 8.11 & Up \\
\hline RP14130014 & Slcola2 & 3.20 & 3.40 & 3.96 & Up \\
\hline RP14131422 & Plag1 & 3.24 & 3.72 & 4.16 & Up \\
\hline RP14132603 & Cenc & 3.17 & 4.21 & 9.26 & Up \\
\hline RP14169885 & Naca & 4.46 & 5.24 & 6.78 & Up \\
\hline RP14170272 & Slc39a5 & 5.49 & 6.28 & 7.65 & Up \\
\hline RP14175180 & Olr1084 & 3.14 & 3.22 & 3.42 & Up \\
\hline RP14175183 & Olr1085 & 3.97 & 4.65 & 4.98 & $\mathrm{Up}$ \\
\hline RP14183524 & Jrk & 3.15 & 4.97 & 7.04 & $\mathrm{Up}$ \\
\hline
\end{tabular}




\begin{tabular}{lccccc}
\hline RP14207074 & Myo5a & 3.37 & 4.16 & 4.77 & $\mathrm{Up}$ \\
RP14211530 & Traip & 3.45 & 5.65 & 6.02 & $\mathrm{Up}$ \\
RP14222274 & Crygb & 3.80 & 5.65 & 6.50 & $\mathrm{Up}$ \\
RP14235002 & Olr1389 & 3.38 & 3.57 & 5.12 & $\mathrm{Up}$ \\
RP14271990 & Ccz1 & 3.69 & 3.98 & 5.10 & $\mathrm{Up}$ \\
RP14279738 & Pla2g1b & 3.73 & 4.07 & 4.56 & $\mathrm{Up}$ \\
RP14288987 & Usf1 & 3.09 & 3.16 & 3.85 & $\mathrm{Up}$ \\
RP14301839 & Inpp5j & 3.49 & 7.37 & 8.39 & $\mathrm{Up}$ \\
RP14329375 & Ddx41 & 3.38 & 6.09 & 6.58 & $\mathrm{Up}$ \\
RP14335189 & Psma2 & 3.35 & 3.78 & 3.78 & $\mathrm{Up}$ \\
RP14338669 & Fundc2 & 4.84 & 8.01 & 10.45 & $\mathrm{Up}$ \\
RP14344056 & Dtwd2 & 3.03 & 6.60 & 10.47 & $\mathrm{Up}$ \\
RP14344660 & Slc12a2 & 3.32 & 4.88 & 6.02 & $\mathrm{Up}$ \\
RP14359583 & Olr1696 & 3.46 & 5.37 & 6.49 & $\mathrm{Up}$ \\
\hline
\end{tabular}

2 


\section{Table 4 (on next page)}

The number of differentially expressed genes (DEGs)in three hexanal exposure group with 1.5 -fold change cutoff and $p$-value $<0.05$ ).

The number of differentially expressed genes (DEGs) in three hexanal exposure group with 1.5 -fold change cutoff and $p$-value $<0.05$ ). 
1 Table 4. The number of differentially expressed genes (DEGs) in three hexanal exposure group with 1.5-fold change cutoff and p-value $<0.05$ ).

\begin{tabular}{cccc}
\hline & Up-regulated genes & Down-regulated genes & Total genes \\
\hline $\begin{array}{c}\text { Low dose } \\
(\mathbf{6 0 0} \mathbf{~ p p m})\end{array}$ & 73 & 570 & 643 \\
\hline $\begin{array}{c}\text { Middle dose } \\
(\mathbf{1 , 0 0 0} \mathbf{~ p m )})\end{array}$ & 600 & 211 & 811 \\
\hline $\begin{array}{c}\text { High dose } \\
(\mathbf{1 , 5 0 0} \mathbf{~ p m )})\end{array}$ & 359 & 210 & 569 \\
\hline
\end{tabular}




\section{Table 5 (on next page)}

GO (Gene Ontology) analysis oftarget genes show that the key biological processes under hexanal inhalationexposure ( $p$-value $<0.05$ ).

GO (Gene Ontology) analysis of target genes show that the key biological processes under hexanal inhalation exposure ( $p$-value $<0.05$ ). 
1 Table 6. GO (Gene Ontology) analysis of target genes show that the key biological processes under hexanal inhalation exposure (p-value $<0.05$ ).

\begin{tabular}{|c|c|c|c|c|}
\hline $\begin{array}{l}\text { GO Accession } \\
\text { No. }\end{array}$ & GO Term & Count & p-value & Genes \\
\hline GO:0007595 & Lactation & 4 & 0.001 & $\begin{array}{l}\text { NM_013197(ALAS2), NM_012630(PRLR), } \\
\text { NM_001012027(SERPINC1), NM_001013248(FOXB1) }\end{array}$ \\
\hline GO:0035914 & Skeletal muscle cell differentiation & 3 & 0.015 & NM_017259(BTG2), NM_024388(NR4A1), NM_013220(ANKRD1) \\
\hline GO:0051965 & $\begin{array}{l}\text { Positive regulation of synapse } \\
\text { assembly }\end{array}$ & 3 & 0.023 & $\begin{array}{l}\text { NM_134376(CLSTN3), NM_001109430(LRTM2), } \\
\text { NM_012892(ASIC2) }\end{array}$ \\
\hline GO:0006814 & Sodium ion transport & 3 & 0.037 & $\begin{array}{l}\text { NM_001113335(SLC9A2), NM_012892(ASIC2), } \\
\text { NM_001109385(SLC9B2) }\end{array}$ \\
\hline GO:0009612 & Response to mechanical stimulus & 3 & 0.037 & NM_017259(BTG2), NM_012892(ASIC2), NM_021836(JUNB) \\
\hline GO:0032680 & $\begin{array}{l}\text { Regulation of tumor necrosis factor } \\
\text { production }\end{array}$ & 2 & 0.025 & NM_133290(ZFP36), NM_001106864 (LTF) \\
\hline GO:0060213 & $\begin{array}{l}\text { Positive regulation of nuclear- } \\
\text { transcribed mRNA poly(A) tail } \\
\text { shortening }\end{array}$ & 2 & 0.025 & NM_133290(ZFP36), NM_017259(BTG2) \\
\hline
\end{tabular}




\section{Table 6(on next page)}

The number of correlated target genes between DNA methylation and mRNAexpression by hexanal exposure ( $p$-value $<0.05$ ).

The number of correlated target genes between DNA methylation and mRNA expression by hexanal exposure ( $p$-value $<0.05)$. 
1 Table 5. The number of correlated target genes between DNA methylation and mRNA expression by hexanal exposure (p-value $<0.05)$.

\begin{tabular}{ccc}
\hline & $\begin{array}{c}\text { Hyper-methylated } \\
\text { vs. } \\
\text { Down-regulated }\end{array}$ & $\begin{array}{c}\text { Hypo-methylated } \\
\text { vs. } \\
\text { Up-regulated }\end{array}$ \\
\hline Low dose (600 ppm) & 7 & 0 \\
Middle dose (1,000 ppm) & 24 & 25 \\
High dose (1,500 ppm) & 44 & 28 \\
\hline
\end{tabular}

